\title{
Die Grundzüge des Designrechts in Deutschland
}

\author{
Philipp Steichele
}

\section{Einleitung}

Das Designrecht bzw. Geschmacksmusterrecht schützt die ästhetisch wirkenden Gestaltungsformen eines Produkts. ${ }^{1}$ Dabei soll der Designschutz zur Innovation und Entwicklung neuer Erzeugnisse sowie zu Investitionen für ihre Herstellung ermutigen. ${ }^{2}$ Gesetzgebungspolitische Motivation des Schutzes für das eingetragene Design und Gemeinschaftsgeschmacksmuster ist es daher, einen wirtschaftlichen Anreiz für Innovationen der Entwerfer und für Investitionen der Produzenten von Erzeugnissen zu schaffen, die die Erfordernisse der Neuheit und Eigenart erfüllen. Der Schutzgegenstand liegt beim Geschmacksmuster bzw. Design - wie bei allen Teilgebieten des gewerblichen Rechtsschutzes - auf dem Schutz immaterieller Güter. Keinen Schutz gewährt das Designrecht für formlose Erzeugnisse wie Cremes oder Flüssigkeiten ohne ein besonderes Erscheinungsbild in der Farbgebung, ${ }^{3} \mathrm{Com}$ puterprogramme, ${ }^{4}$ Tiere oder Pflanzen, ${ }^{5}$ Ideen oder allgemeine Gedanken.

Das Geschmacksmuster - heute Designrecht - ist Teil des Immaterialgüterrechts, ${ }^{6}$ das sich insgesamt mit dem Schutz nicht gegenständlicher, geistiger Leistungen befasst. Oft ist der Erfolg eines Produkts auch abhängig von dessen

\footnotetext{
${ }^{1}$ Eichmann, Designrecht, in: Eichmann/Kur, Praxishandbuch ${ }^{2}$ (2016) §§ 1 Rn. 1.

${ }^{2}$ Erwägungsgrund 7 der Verordnung (EG) Nr. 6/2002 des Rates vom 12. Dezember 2001 über das Gemeinschaftsgeschmacksmuster.

${ }^{3}$ Eichmann, in: Eichmann/Kur, $§ 2$ Rn. 24 f.

${ }^{4}$ Eichmann, in: Eichmann/Kur, §§ 2 Rn. 10.

${ }^{5}$ Eichmann, in: Eichmann/Kur, §§ 2 Rn. 40.

${ }^{6}$ Kunze, Das neue Geschmacksmusterrecht: Einführung, Texte, Materialien (2004), S. 11.
}

\author{
P. Steichele $(\square)$ \\ h.d. steichele - Rechtsanwälte, München, Deutschland \\ E-Mail: phsteichele@hdsteichele.de
}


Design, d. h. von seiner Form, Farbe und ästhetischen Gestaltung, weshalb der Produktaufmachung für die Vermarktung eine erhebliche Bedeutung zukommt. Dies gilt in vielen Bereichen, wie etwa bei dem Design von Autos, Möbelstücken, Haushaltsgeräten, Kinderspielzeug, Sportschuhen, Handyschutzhüllen, Staubsauger etc. Das Design beeinflusst die Kaufentscheidung des Verbrauchers mit unter ganz wesentlich.

\section{Allgemeine Grundlagen}

\subsection{Historische Entwicklung und rechtliche Grundlagen}

Das Geschmacksmuster- bzw. Designrecht als ältestes gewerbliches Schutzrecht in Deutschland hat seinen Ursprung im Mittelalter. Zünfte gewährten dem Erwerber eines neuen Musters für ästhetische Gestaltungsformen von Waren einen zeitlich begrenzten Schutz gegen Nachbildungen. ${ }^{7}$ In Deutschland kam ein gesetzlicher Musterschutz erstmals 1806 in den Rheinlanden durch die französische Gesetzgebung auf, die allgemeine Einführung erfolgte aber erstmals durch das Reichsgesetz vom 11. Januar 1876 betreffend das Urheberrecht an Mustern und Modellen. ${ }^{8}$ Das „Geschmacksmuster“, welches auf das Jahr 1876 zurückgeht, ${ }^{9}$ ist das älteste gewerbliche Schutzrecht in Deutschland. Gewerbliche Schutzrechte sind allgemein Rechte zum Schutz geistiger Ideen, Schutz vor Nachahmung und eigenen kreativen Leistungen; zu den gewerblichen Schutzrechten zählen auch das Patentrecht und das Markenrecht, nicht hingegen das Urheberrecht: Den gewerblichen Schutzrechten ist gemein, dass sie in ein Register eingetragen werden; das Urheberrecht entsteht hingegen automatisch, „kraft Schöpfung“. Am 1. Juni 2004 trat das deutsche Gesetz über den rechtlichen Schutz von Mustern und Modellen - Geschmacksmustergesetz - in Kraft. ${ }^{10}$ Damit wurde das über 125 Jahre alte deutsche Geschmacksmustergesetz von 1876 durch ein neues, modernes und eigenständiges gewerbliches Schutzrecht ${ }^{11}$ abgelöst, mit dem die Richtlinie des Europäischen Parlaments und Rates 98/71/EG vom 13.10.1998 über den rechtlichen Schutz von Mustern und Modellen umgesetzt wurde. ${ }^{12}$ Bis zum Inkrafttreten des neuen Geschmacksmustergesetzes (GeschmMG) am 1. Juni 2004 kam dem Geschmacksmuster eine „Zwitterstellung“" zwischen Urheberrecht und den gewerblichen Schutzrechten zu. ${ }^{13}$ Das

\footnotetext{
${ }^{7}$ Nirk, Geschmacksmusterrecht, Urheberrecht und Designlaw (2010), S. 4.

${ }^{8}$ Nirk 2010, S. 4; eingehend zur historischen Entwicklung des Designschutzes im deutschen und europäischen Recht Eichmann, in: Eichmann/Kühne, A. Allgemeines zum Designrecht Rn. 2 ff.; Polater 2013, S. $6 \mathrm{ff}$.

${ }^{9}$ Vgl. BT-Drucks. 15/1075.

${ }^{10}$ BGBl. I 2004, 390.

${ }^{11}$ Vgl. auch BGH, NJW 2014, 469 (473).

${ }^{12}$ BT-Drucks. 15/1075, S. 26.

${ }^{13}$ Kunze 2004, S. 11.
} 
Geschmacksmustergesetz brachte die wesentliche Neuerung, dass es sich bei einem Geschmacksmuster ab Eintragung mit Bildbekanntmachung nunmehr um ein umfassendes Verbietungsrecht mit absoluter Sperrwirkung handelte. ${ }^{14}$ Schließlich wurde das deutsche Geschmacksmustergesetz mit Wirkung zum 01.01.2014 durch das Gesetz über den rechtlichen Schutz von Design (Designgesetz - DesignG) abgelöst, das neben einer terminologischen Neuausrichtung des Begriffs „Geschmacksmuster", der fortan durch den Begriff ,Design“ ersetzt wurde,${ }^{15}$ die Einführung eines Nichtigkeitsverfahrens vor dem Deutschen Patent- und Markenamt (DPMA) sowie Neuregelungen zur Geltendmachung der Rechtsungültigkeit eines Geschmacksmusters bzw. Designs mit sich brachte. Das DesignG wird flankiert von der Verordnung zur Ausführung des Designgesetzes (DesignV), die u. a. Regelungen zum Eintragungsverfahren, Inhalt des Designregisters, Internationalen Eintragungen sowie Ergänzungen zum Nichtigkeitsverfahren enthält. Neben dem nationalen Designschutz wird durch das europäische (Gemeinschafts-) Geschmacksmusterrecht Musterschutz jenseits nationaler Grenzen innerhalb der EU gewährleistet. Am 6. März 2002 trat die am 5. Januar 2002 veröffentlichte Gemeinschaftsgeschmacksmusterverordnung (EG) Nr. 6/2002 - kurz GGV - in Kraft, mit der ein System für die Erlangung eines Gemeinschaftsgeschmacksmusters mit einheitlichem Schutz und einheitlicher Wirkung für die gesamte europäische Gemeinschaft geschaffen wurde. Durch ein EU-Geschmacksmuster wurde folglich die Möglichkeit geschaffen, mit einer Anmeldung binnen kürzester Zeit Schutz in derzeit 28 Mitgliedstaaten zu erlangen.

\subsection{Designschutz auf drei Ebenen}

Der Schutz ästhetischer Leistungen erfolgt auf drei Stufen: Auf nationaler Ebene wird der Designschutz in Deutschland durch die Eintragung beim Deutschen Patent- und Markenamt erreicht, wobei die Schutzwirkung gem. § 27 Abs. 1 DesignG erst mit der Eintragung in das Register entsteht. Die Schutzdauer des eingetragenen Designs erstreckt sich gemäß $§ 27$ Abs. 2 DesignG auf 25 Jahre, beginnend mit dem Tag der Anmeldung. Daneben erfolgt der Schutz des Designs in anderen Mitgliedstaaten der Europäischen Union durch das Gemeinschaftsgeschmacksmuster nach Art. 1 Abs. 1 der Verordnung (EG) Nr. 6/2002 des Rates vom 12. Dezember 2001. Anders als der Designschutz auf nationaler Ebene in Deutschland, der zwingend an die Eintragung beim Deutschen Patent- und Markenamt geknüpft ist, kann Musterschutz beim Gemeinschaftsgeschmacksmuster gemäß Art. 12 Abs. 2 der Verordnung (EG) Nr. 6/2002 entweder durch Eintragung beim Amt der Europäischen Union für geistiges Eigentum (EUIPO) ${ }^{16}$ oder auch formlos als ein nicht eingetrage-

\footnotetext{
${ }^{14}$ Kunze 2004, S. 11.I

${ }^{15}$ Vgl. hierzu BT-Drucks. 17/13428, S. 1 f.

${ }^{16}$ Die frühere, in Art. 2 der Verordnung (EG) Nr. 6/2002 noch enthaltene, Bezeichnung ,Harmonisierungsamt für den Binnenmarkt" wurde durch die Verordnung (EU) 2015/2424 mit Wirkung zum 16.03.2016 abgeändert.
} 
nes Gemeinschaftsgeschmacksmuster entstehen, ${ }^{17}$ wenn Letzteres der Öffentlichkeit so zugänglich gemacht wird, dass innerhalb der EU hiervon Kenntnis erlangt werden kann. Während die Schutzdauer von regulär 5 Jahren für das eingetragene Gemeinschaftsgeschmacksmuster gemäß Art. 12 der Verordnung (EG) Nr. 6/2002 bis zu einer Gesamtlaufzeit von 25 Jahren ab dem Anmeldetag verlängerbar ist, ist die Schutzwirkung des nicht eingetragenen Rechts gemäß Art. 11 der vorstehenden Verordnung auf 3 Jahre ab dem Anmeldetag begrenzt. Darüber hinaus gewährleistet das nach dem Haager Musterabkommen beim Internationalen Büro der Weltorganisation für geistiges Eigentum (WIPO) anzumeldende internationale Geschmacksmuster Designschutz auf dritter, internationaler Ebene. Die Schutzdauer eines internationalen Geschmacksmusters beläuft sich regulär auf 5 Jahre, verlängerbar auf 15 Jahre.

\subsection{Schutzgegenstand des Designrechts}

Als Design definiert $§ 1$ Nr. 1 DesignG die zweidimensionale oder dreidimensionale Erscheinungsform eines ganzen Erzeugnisses oder eines Teils davon, die sich insbesondere aus den Merkmalen der Linien, Konturen, Farben, der Gestalt, Oberflächenstruktur oder der Werkstoffe des Erzeugnisses selbst oder seiner Verzierung ergibt. Die Aufzählung der Merkmale, die eine Erscheinungsform begründen können, ist beispielhaft und nicht abschließend. Sie umfasst nicht nur visuell wahrnehmbare Wirkungen, sondern auch - anders als nach dem altem deutschen Recht taktile Wirkungen, was insbesondere durch die Aufnahme der Oberflächenstruktur als schutzfähige Erscheinungsform deutlich wird. Auch das Gewicht und die Biegsamkeit können zur Produktgestaltung beitragen; ob es sich dabei jedoch um eine musterfähige Erscheinungsform handelt, wird in der Literatur unterschiedlich beurteilt. ${ }^{18}$ Schwierigkeiten ergeben sich daraus, dass derartige Merkmale regelmäßig nicht das Anmeldeerfordernis der grafischen Darstellbarkeit, vgl. § 11 Abs. 2 Nr. 3 DesignG bzw. Art. 36 Abs. 1 lit. c) GGV erfüllen; insoweit bleibt aber der Schutz als nicht eingetragenes Gemeinschaftsgeschmacksmuster möglich. Begriffe wie „Vorbild“, ,ästhetische Wirkung“, wie sie aus der früheren deutschen Gesetzgebung und Rechtsprechung zur Musterfähigkeit bekannt sind, spielen heute keine Rolle mehr. Für die Zugänglichkeit zum Design- bzw. Geschmacksmusterschutz genügt die bloße visuelle oder taktile Wahrnehmbarkeit. Weitere Grundvoraussetzung für den Design- bzw. Geschmacksmusterschutz ist das Vorhandensein eines Erzeugnisses gemäß § 1 Nr. 2 DesignG bzw. Art. 3 lit. b) GGV, worunter jeder industrielle oder handwerkliche Gegenstand verstanden wird, einschließlich Verpackungen, Ausstattungen, grafischer Symbole und typografischer Schriftzeichen sowie

\footnotetext{
${ }^{17}$ Vgl. Kunze 2004, S. 14.

${ }^{18}$ Polater, Designschutz nach dem deutschen, europäischen und türkischen Geschmacksmusterrecht (2013), S. $37 \mathrm{ff}$.
} 
Einzelteile, die zu einem komplexen Erzeugnis zusammengebaut werden können. ${ }^{19}$ Fehlt dem der Erscheinungsform zugrunde liegenden Gegenstand die Eigenschaft als Erzeugnis, besteht kein Schutz. ${ }^{20}$ Damit ist das Vorliegen eines Erzeugnisses zwar Voraussetzung für den Designschutz, aber das Erzeugnis selbst nicht Schutzobjekt, sondern dessen Erscheinungsform. ${ }^{21}$ Die in $\S 1$ Nr. 2 DesignG bzw. Art. 3 lit. b) GGV genannten Beispiele machen deutlich, dass der Erzeugnisbegriff weit auszulegen ist. Die Gegenstände, die industriell oder handwerklich gefertigt sein müssen, können ein- oder mehrteilig sein. Insofern kann die Erscheinungsform eines Teils eines Erzeugnisses (Einzelteil oder Bauelement) ein schutzfähiges Design bzw. Geschmacksmuster begründen, ${ }^{22}$ unabhängig davon, ob das Teil wesentlicher Bestandteil des Gesamterzeugnisses ist oder nicht. Zur Frage des Schutzgegenstands des Designrechts unter der Wiedergabe unterschiedlicher Darstellungen bei der Anmeldung hat der Bundesgerichtshof im Rahmen seiner ,Weinkaraffen“-Entscheidung vom 08.03.2012 zudem klargestellt, dass auch dann, wenn die Anmeldung eines nationalen Designs wie auch eines Gemeinschaftsgeschmacksmusters mehrere Darstellungen der Erscheinungsform enthält, diese nur einen einzigen Schutzgegenstand bilden. ${ }^{23}$ Der Schutzgegenstand kann demnach auch aus mehreren Gegenständen bestehen, die nach der Verkehrsauffassung als ein einheitliches sog. Kombinationserzeugnis anzusehen sind. ${ }^{24}$

\section{Materielle Schutzvoraussetzungen}

Voraussetzung für den Schutz eines eingetragenen Designs sowie für den Schutz als Gemeinschaftsgeschmacksmuster sind nach § 2 Abs. 1 DesignG bzw. Art. 4 Abs. 1 GGV die Neuheit und Eigenart des Designs bzw. Musters. Die Schutzvoraussetzungen sind für das eingetragene Design und Gemeinschaftsgeschmacksmuster insoweit folglich gleich ausgestaltet und waren in Deutschland bereits im ersten Geschmacksmustergesetz von 1876 als Kernvoraussetzungen enthalten. ${ }^{25}$ Eine darüber hinaus gehende gewerbliche Verwertbarkeit ist hingegen ebenso wenig Voraussetzung wie ein Benutzungszwang. ${ }^{26}$

\footnotetext{
${ }^{19}$ Polater 2013, S. 40 f.; Wöhrn, Medienrecht: Praxishandbuch, Teil 2, Kapitel 11 Rn. 16.

${ }^{20}$ So z. B. bei Computerprogrammen (siehe $\S \S 1$ Nr. 2 DesignG letzter Halbsatz). Auch Farben und Gerüche als solche, stellen, ohne auf ein konkretes Erzeugnis gerichtet zu sein, keine schutzfähigen Erzeugnisse dar; vgl. Eichmann, in: Eichmann/Kur, §§ 2 Rn. 24, 27.

${ }^{21}$ Eichmann, in: Eichmann/Kur, $\S \S 2$ Rn. 10.

${ }^{22}$ Eichmann, in: Eichmann/Kur, $\S § 2$ Rn. 8.

${ }^{23} \mathrm{BGH}$, Urteil vom 08.03.2012 - I ZR 124/10, Rn 17 ff. (juris).

${ }^{24}$ BGH, Urteil vom 08.03.2012 - I ZR 124/10, Rn 32 (juris); Eichmann, in: Eichmann/Kur, $\S 2$ Rn. 15.

${ }^{25}$ Eichmann, in: Eichmann/Kur, $\S 2$ Rn. 62.

${ }^{26}$ Nirk 2010, S. 25.
} 


\subsection{Neuheit}

Als neu gilt ein Design nach $§ 2$ Abs. 2 DesignG, wenn vor dem Anmeldetag kein identisches Design offenbart, d. h. nach $\S 5$ DesignG ausgestellt, veröffentlicht oder in sonstiger Form auf den Markt gebracht worden ist, wobei ein Design gem. § 2 Abs. 2 S. 2 DesignG dann als identisch anzusehen ist, wenn sich ihre Merkmale nur in unwesentlichen Einzelheiten unterscheiden. ${ }^{27}$ Maßgeblich ist die objektiv-relative Neuheit eines Musters. ${ }^{28}$ Für die Neuheit eines Designs genügen demnach bereits kleine Detailveränderungen, während reine Kopien und Gestaltungen mit nur abweichenden Abmessungen dem neuheitschädlichen Bereich zuzuordnen sind. ${ }^{29}$ Die Beurteilung der Neuheit erfolgt durch einen Einzelvergleich zwischen dem zu prüfenden und bekannten Design. ${ }^{30}$ Was als neu zu bewerten ist, bestimmt sich danach, ob die prägenden und die die Eigenart begründenden Gestaltungselemente den einschlägigen inländischen Fachkreise innerhalb der betreffenden Wirtschaftszweige im Anmeldezeitpunkt bereits bekannt waren oder bei zumutbarer Beachtung der auf dem Gebiet vorhandenen Gestaltungen bekannt sein konnten. ${ }^{31}$ Eine Veröffentlichung ist dann als den Fachkreisen nicht bekannt anzusehen, wenn sie bereits vor so langer Zeit oder an einem so entfernt liegenden oder unzugänglichen Ort erfolgte, dass sie nicht berücksichtigt werden konnte.

\subsection{Eigenart}

Zweite Voraussetzung für die Schutzfähigkeit ist die die frühere Eigentümlichkeit ersetzende Eigenart des Designs. ${ }^{32}$ Als eigentümlich bewertete der Bundesgerichtshof ein Muster oder Modell, wenn ,es in den für die ästhetische Wirkung maßgebenden Merkmalen als das Ergebnis einer eigenpersönlichen, form- oder farbenschöpferischen Tätigkeit erscheint" ". ${ }^{33}$ Von dem Erfordernis der Eigentümlichkeit und Gestaltungshöhe wurde mit der Neuformulierung der Eigenart in $\S 2$ Abs. 3 Geschmacksmustergesetz sowie nachfolgend § 2 Abs. 3 DesignG Abstand genommen $^{34}$ und setzt die Eigenart i. S. v. $§ 2$ Abs. 3 DesignG sowie Art. 6 Abs. 1 GGV

\footnotetext{
${ }^{27}$ Vgl. auch Art. 5 Abs. 2 GGV.

${ }^{28}$ BGHZ 50, 340 (344 ff.); Polater 2013, S. 56 m.w.N.; Wöhrn, in: Wandtke, Teil 2, Kapitel 11 Rn. 28.

${ }^{29}$ Gottschalk, Der Schutz des Designs nach deutschem und europäischen Recht (2005), S. 64 m. w. N.

${ }^{30}$ Engels, Patent-, Marken- und Urheberrecht, Leitfaden für Ausbildung und Praxis (2015), Kapitel 5 Rn. 1025.

${ }^{31}$ BGHZ 50, 340 (355 f.); Wöhrn, in: Wandtke, Teil 2, Kapitel 11 Rn. 28.

${ }^{32}$ Zur „Eigentümlichkeit“ nach früherem Recht vgl. §§ 1 Abs. 2 des Gesetzes betreffend das Urheberrecht an Mustern und Modellen vom 11.01.1876, RGBl 1876, S. 11.

${ }^{33}$ BGH, NJW 1968, 2193 (2198).

${ }^{34}$ Engels 2015, Kapitel 5 Rn. 1026b; Kunze 2004, S. 18; zum Gemeinschaftsgeschmacksmuster BGHZ 185, $224 \mathrm{ff.}$
} 
nunmehr allein voraus, dass sich der Gesamteindruck, den ein Design beim informierten Benutzer, von dem ein gewisses Maß an Designbewusstsein verlangt wird, ${ }^{35}$ hervorruft, von dem Gesamteindruck unterscheidet, den ein anderes Design im Einzelvergleich bei diesem Benutzer hervorruft, das vor dem Anmeldetag offenbart worden ist. ${ }^{36}$ Zur Beurteilung, ob eine ausreichende Eigenart gegeben ist, ist gem. $\S 2$ Abs. 3 S. 2 DesignG, Art. 6 Abs. 2 GGV der Grad der Gestaltungsfreiheit des Entwerfers bei der Entwicklung des Musters zu berücksichtigen. ${ }^{37}$ Zwischen dem Gestaltungsspielraum des Entwerfers und dem Gesamteindruck eines Musters besteht damit eine zu beachtende Wechselwirkung. ${ }^{38}$ Dies hat zur Folge, dass bereits geringe Gestaltungsunterschiede im Kreise des informierten Benutzers einen anderen Gesamteindruck hervorrufen können, während umgekehrt bei einer geringen Musterdichte ein weiterer Schutzumfang eines Musters besteht und sogar größere Gestaltungsunterschiede unter Umständen keinen anderen Gesamteindruck erwecken. ${ }^{39}$ Je höher demnach die Zahl der bekannten Muster auf einem bestimmten Gebiet ist, desto geringer sind die an die Eigenart zu stellenden Anforderungen. Anders gewendet: bei einer geringen Zahl von vorbekannten Mustern wirkt sich ein Begeben in die Nähe des vorbekannten Formenschatzes deshalb nachteilig aus, weil der informierte Benutzer in diesem Fall eher eine Übereinstimmung der Gesamteindrücke bejahen wird. Existieren in einem Bereich nur wenige bekannte Designs, muss der Gestaltungsabstand in der Regel größer sein, als in Bereichen, in denen eine hohe Designdichte besteht.

\subsection{Neuheitsschonfrist}

Um dem Entwerfer die Möglichkeit zu geben vor der Anmeldung sein Design der Öffentlichkeit zugänglich zu machen und seine Marktchancen auszuloten, ${ }^{40}$ enthält $\S 6$ DesignG eine 12-monatige Neuheitsschonfrist. Danach bleibt eine Offenbarung bei der Prüfung der Neuheit und Eigenart außer Betracht, wenn ein Design während der 12 Monate vor dem Anmeldetag oder für den Fall, dass eine Priorität nach $\S \S 14,15$ DesignG in Anspruch genommen wird, ab dem Prioritätstag gem. § 13 Abs. 2 DesignG, durch den Entwerfer, seinen Rechtsnachfolger oder durch einen Dritten als Folge der Öffentlichkeit zugänglich gemacht wird. Während der Schonfrist kann das Design folglich ohne nachteilige Folgen für die Eintragung vom

\footnotetext{
${ }^{35}$ Eichmann, in: Eichmann/Kühne, Designgesetz, Gesetz über den rechtlichen Schutz von Design, $\S \S 2$ Rn. 29.

${ }^{36}$ Engels 2015, Kapitel 5 Rn. 1026; Kunze 2004, S. 18.

${ }^{37}$ Engels 2015, Rn. 1027; zum erforderlichen Grad der Unterschiedlichkeit Gottschalk 2005, S. 72; Wöhrn, in: Wandtke, Teil 2, Kapitel 11 Rn. 21 ff.

${ }^{38}$ BGH, Urt. vom 19.05.2010 - I ZR 71/08 Rn. 17 (juris); Eichmann, in: Eichmann/Kur, §§ 2 Rn. 72.

${ }^{39} \mathrm{BGH}$, Urt. vom 19.05.2010 - I ZR 71/08 Rn. 17 (juris).

${ }^{40}$ Gottschalk 2004, S. 67; Erwägungsgrund 20 GGV.
} 
Entwerfer benutzt oder in sonstiger Weise der Öffentlichkeit zugänglich gemacht werden. ${ }^{41}$ Anders als dem Patentrecht liegt dem Designschutz damit ein relativer Neuheitsbegriff zugrunde, der einen Schutz auch dann gewährleistet, wenn der Schutzgegenstand der Öffentlichkeit zuvor für einen Maximalzeitraum von 12 Monaten bekannt war.

\subsection{Kein Schutzausschluss}

Bestimmte Erscheinungsmerkmale und Designs sind aufgrund technischer oder moralischer Aspekte vom Designschutz nach § 3 DesignG ausgenommen. Ausgeschlossen vom Designschutz sind zum einen Erscheinungsmerkmale eines Erzeugnisses, die ausschließlich durch deren technische Funktion bedingt sind, zum anderen Gestaltungsformen, die funktionell bedingt sind und zwangsläufig eine bestimmte Form aufweisen müssen (sog. „,must-fit"-Teile). ${ }^{42}$ Diese funktionsbedingten Vorgaben, etwa bei einer Toilettenbrille, sind ganz allgemein gattungsspezifische Merkmale. Weiterhin ist gem. § 3 Abs. 1 Nr. 3 und 4 DesignG auch solchen Gestaltungen, die gegen die öffentliche Ordnung oder gegen die guten Sitten verstoßen, kein Schutz zuzubilligen, wobei hierunter insbesondere sexualbezogene und herabwürdigende pornografische Darstellungen fallen. ${ }^{43}$

\section{Eintragung und Schutzdauer des deutschen Designs}

Als Formalrecht ist das Designrecht beim deutschen Patent- und Markenamt (DPMA) eintragungspflichtig. Erst mit der Eintragung in das dort geführte Designregister entsteht nach $\S 27$ Abs. 1 DesignG der Schutz des Designs. Anders als das Markenrecht kann das eingetragene Designrecht nicht allein kraft Verkehrsdurchsetzung begründet werden. Besonderheiten ergeben sich insoweit beim Gemeinschaftsgeschmacksmuster gem. Art. 1 Abs. 1 GGV, da dieses als nicht eingetragenes Gemeinschaftsgeschmacksmuster nach Art. 1 Abs. 2 b) GGV Schutzwirkung bereits ab dem Zeitpunkt entfaltet, zu dem es der Öffentlichkeit in der Gemeinschaft zugänglich gemacht wird. Im Unterschied zum eingetragenen Gemeinschaftsgeschmacksmuster ist der formlose Schutz nach Art. 19 Abs. 2 GGV allerdings auf die Abwehr von Nachahmungen als bewusste bzw. gezielte Nachbildung beschränkt. ${ }^{44}$

\footnotetext{
${ }^{41}$ Gottschalk 2004, S. 67.

${ }^{42}$ Kunze 2004, S. 18.

${ }^{43}$ Vgl. Eichmann, in: Eichmann/Kur, §§ 2 Rn. 95; Polater 2013, S. 100.

${ }^{44}$ Hierzu eingehend Gottschalk 2005, S. 89 ff.
} 
Auch wenn das deutsche Recht einen solchen formlosen Schutz nicht eingetragener Designs nicht kennt, gewährt der Bundesgerichtshof in ständiger Rechtsprechung für ein nicht eingetragenes Design unter bestimmten Umständen aber ergänzenden wettbewerbsrechtlichen Leistungsschutz nach dem Gesetz gegen den unlauteren Wettbewerb (UWG). Dieser ermöglicht es, gegen den Vertrieb von Produkten vorzugehen, die eine vermeidbare Herkunftstäuschung hervorrufen oder den guten Ruf oder die Wertschätzung des Originalprodukts beeinträchtigen. Voraussetzung ist, dass das Originalprodukt über wettbewerbliche Eigenart verfügt. Die Gestaltung des Produkts muss insoweit die Eignung besitzen, auf seine Herkunft hinzuweisen. Für den nationalen Designschutz beläuft sich die reguläre Schutzdauer auf 5 Jahre, berechnet ab dem Anmeldetag ${ }^{45}$ und kann nach $\S 27$ Abs. 2, 28 Abs. 1 DesignG auf eine maximale Schutzdauer von 25 Jahren ausgeweitet werden. Der Beginn des Schutzes und der für die Berechnung der Laufzeit maßgebliche Zeitpunkt (Anmeldetag) fallen folglich vergleichbar mit der Rechtslage im Patent- und Markenrecht auseinander. $^{46}$

Von zentraler Bedeutung für die Festlegung des Prüfungsumfangs durch das Deutsche Patent- und Markenamt ist die Einordnung des Design- bzw. Geschmacksmusterrecht als sog. „Registrierrecht“" ${ }^{47}$ Danach nimmt das Deutsche Patent- und Markenamt keine Sachprüfung vor, sondern prüft gem. § 16 DesignG bei der Eintragung des Designs - gleiches gilt für das Gemeinschaftsgeschmacksmuster - ausschließlich die formellen Voraussetzungen einer Eintragung, wozu insbesondere die Designfähigkeit des angemeldeten Designs sowie die Wahrung der Erfordernisse nach der Designverordnung gehören. Nicht geprüft werden die materiellen Schutzvoraussetzungen in Gestalt der Neuheit und Eigenart des Designs sowie ein möglicherweise eingreifender Schutzausschluss nach $\S 3$ Abs. 1 Nr. 1 und 2 DesignG. ${ }^{48}$ Ist Gegenstand der Anmeldung kein Design oder liegt ein Ausschlussgrund nach $\S 3$ Abs. 1 Nr. 3, 4 DesignG vor, etwa wegen Verstoßes gegen die guten Sitten oder der missbräuchlichen Verwendung staatlicher Hoheitszeichen, weist das Deutsche Patent- und Markenamt die Anmeldung gem. § 18 DesignG zurück. Resultierend aus der fehlenden materiellen Prüfungskompetenz des Deutschen Patent- und Markenamtes führt das Designrecht auch die Bezeichnung ,ungeprüftes Schutzrecht““.99 Fehlt es an den Schutzvoraussetzungen nach $§ 2$ DesignG, entsteht trotz der formellen Eintragung kein Schutzrecht.

\footnotetext{
${ }^{45}$ BT-Drucks. 15/1075, S. 46.

${ }^{46}$ BT-Drucks. 15/1075, S. 46; zur Schutzdauer des eingetragenen Gemeinschaftsgeschmacksmusters Art. 12 GGV.

${ }^{47}$ BT-Drucks. 15/1075, S. 30.

${ }^{48}$ BT-Drucks. 15/1075, S. 30.

${ }^{49}$ Wöhrn, in: Wandtke, Teil 2, Kapitel 11 Rn. 49.
} 


\section{Schutzumfang und Folgen der Schutzverletzung}

\subsection{Wirkungen des eingetragenen Schutzrechtes}

Das eingetragene Design - ebenso das eingetragene Gemeinschaftsgeschmacksmuster - gewähren dem Rechtsinhaber das ausschließliche Recht, Dritten zu verbieten, das Schutzgut ohne seine Zustimmung zu benutzen, wozu gem. $§ 38$ Abs. 1 DesignG sowohl die Herstellung als auch das Anbieten, Inverkehrbringen, der Im- und Export sowie die Ausstellung, der Gebrauch eines Erzeugnisses, in welches das Design aufgenommen wurde, und der Besitz eines solchen Erzeugnisses zu den genannten Zwecken gehört. Das Designrecht begründet damit eine gegenüber der früheren Gesetzeslage vor Inkrafttreten des Gesetzes über den rechtlichen Schutz von Mustern und Modellen (Geschmacksmustergesetz) vom 12.03.2004 weitreichendere, absolute Sperrwirkung. ${ }^{50}$ Beschränkungen des Rechts aus dem eingetragenen Design ergeben sich lediglich aus $\S \S 40,41$ DesignG.

\subsection{Rechte aus dem Design}

Mit der Ausgestaltung des Designrechts als Recht mit absoluter Sperrwirkung korrespondiert für den Entwerfer wie auch dessen Rechtsnachfolger ${ }^{51}$ als Rechtsinhaber der Schutz vor rechtswidrigen Verletzungen des eingetragenen Designs durch Dritte; gesetzlich verankert in $\S \S 42$ ff. DesignG, Art. 89 GGV. Dem Rechtsinhaber steht neben dem Anspruch auf Beseitigung der Beeinträchtigung, auf Unterlassung oder Vernichtung und Überlassung der rechtswidrig hergestellten bzw. verbreiteten Erzeugnisse bei einer vorsätzlichen oder fahrlässigen Verletzungshandlung zugleich ein Anspruch auf Schadensersatz zu. ${ }^{52}$ Eingeschränkt wird die Geltendmachung zivilrechtlicher Ansprüche durch die Ausschlussregelung in $\S 40$ DesignG, wonach insbesondere Handlungen im privaten Bereich ausgeklammert werden.

\subsection{Abgrenzung zu anderen Schutzrechten}

Die Zuordnung des Designrechts zu den gewerblichen Schutzrechten macht zugleich eine Abgrenzung zum Marken-, Urheber- und Patentrecht erforderlich. Während für das Gemeinschaftsgeschmacksmuster durch Art. 96 Abs. 1 GVV klargestellt

\footnotetext{
${ }^{50}$ BT-Drucks. 15/1075, S. 30.

${ }^{51}$ Vgl. $\S 7$ Abs. 1 S. 1 DesignG; Art. 14 Abs. 1 GGV sowie Eichmann, in: Eichmann/Kur, $\S \S 2$ Rn. 97.

${ }^{52} \mathrm{Zu}$ den möglichen zivilrechtlichen Ansprüchen ausführlich Eichmann, in: Eichmann/Kur, §§ 11 Rn. 2 ff.; Polater 2013, S. 195 ff.
} 
ist, dass Bestimmungen über nicht eingetragene Muster, Marken oder sonstige Zeichen mit Unterscheidungskraft, Patente und Gebrauchsmuster sowie über den unlauteren Wettbewerb unberüht bleiben, hat der deutsche Gesetzgeber dies in $\S 50$ DesignG in allgemeiner Form verankert, wonach Ansprüche aus anderen gesetzlichen Vorschriften unberührt bleiben. ${ }^{53}$

\subsubsection{Markenrecht}

Das Markengesetz regelt den Schutz von Kennzeichen, d. h. Marken, geschäftlichen Bezeichnungen und geografischen Herkunftsangaben, § 1 MarkenG. Als Marke schutzfähig sind z. B. Buchstaben (Textmarken), Zahlen (Zahlenmarken), Bilder (Bildmarken), dreidimensionale Gestaltungen, Firmennamen, Kombinationen aus Wort und Bild. Dem Markenrecht kommt dabei eine betriebliche Unterscheidungsfunktion zu, wonach Waren und Dienstleistungen eines Unternehmens von denen eines anderen Unternehmens zu unterscheiden sein sollen. Ein Geschmacksmuster kann zugleich auch als Marke den Schutz des Markengesetzes erfahren. Da eine Marke vor Verwechslungen schützen soll, ist ihr Schutz auf identische und ähnliche Produkte beschränkt. Bereits bei der Anmeldung einer Marke muss angegeben werden, für welche Waren und Dienstleistungen der Schutz bestehen soll. Für andere Waren und Dienstleistungen ist eine Marke in der Regel nicht geschützt. Ausnahmen gelten für berühmte Marken unter dem Gesichtspunkt der Rufausbeutung. Einerseits kann der Markenschutz theoretisch unendlich lange verlängert werden, andererseits korrespondiert damit eine Pflicht zur Benutzung der Marke, da es keine unnötige Monopolisierung von Kennzeichen geben soll, wenn keine Produkte existieren, die vor einer Verwechslung geschützt werden müssen. Wird eine Marke länger als fünf Jahre für alle oder einige der angegebenen Waren und Dienstleistungen nicht benutzt, wird die Marke für die nicht genutzten Waren und Dienstleistungen auf Antrag eines jeden gelöscht. Ein Geschmacksmuster schützt unabhängig von einem konkreten Produkt vor Nachahmungen. Ob ein Grafikdesign als Firmenlogo, T-Shirt-Aufdruck oder zur Illustration in Büchern benutzt wird, ist unerheblich. Der Schutz gilt generell. Das eingetragene Geschmacksmuster kann Schutz sogar gegen zufällig in Unkenntnis des älteren Geschmacksmusters entwickelte jüngere Designs beanspruchen. Das nicht eingetragene Gemeinschaftsgeschmacksmuster schützt dagegen nur gegen bewusste Nachahmungen, was im Streitfall zu einem Problem führen kann.

\subsubsection{Urheberrecht}

Die Art und Weise wie etwas gestaltet und wahrnehmbar gemacht wird, kann neben dem Designschutz oder Markenschutz auch den Urheberschutz begründen. Im Urheberrecht geht es gem. $§ 2$ Abs. 2 UrhG um den Schutz der persönlichen geistigen

${ }^{53}$ Vgl. Eichmann, in: Eichmann/Kur, §§ 2 Rn. 181. 
Schöpfung und somit um Aspekte der künstlerischen Gestaltung eines Werkes. Designprodukte fallen in den Bereich der angewandten Kunst, die zu den Werken der bildenden Kunst zählen und nach $§ 2$ Abs. 1 Nr. 4 UrhG Urheberschutz genießen. Der Urheberschutz bietet gegenüber dem Design- und Markenschutz den Vorteil, dass er formlos und ohne Gebühren, alleine durch Erschaffen des Werkes entsteht und seine Schutzwirkung nicht erst nach erfolgreicher Registrierung entfaltet. Im Verhältnis zum Designschutz ist die urheberrechtliche Schutzdauer länger; die Schutzdauer lässt sich zwar nicht wie im Markenrecht beliebig durch Zahlung von Gebühren verlängern, endet aber erst 70 Jahre post mortem nach dem Ableben des Urhebers. ${ }^{54}$ Der Urheberschutz setzt anders als Designschutz, für den allein Neuheit und Eigenart entscheidend sind, aber stets ein gewisses Maß an Individualität voraus, das Werk muss einen ,,hinreichend schöpferischen Eigentümlichkeitsgrad““ 55 „Originalität“" ${ }^{56}$ „,eine Schöpfungs- oder Werkhöhe“ ${ }^{\text {"57 }}$ aufweisen. In der Vergangenheit entsprach es gefestigter deutscher Rechtsprechung, dass an den urheberrechtlichen Schutz von Industriedesigns wie etwa Möbeln, Lampen oder Produktverpackungen hohe Anforderungen $\mathrm{zu}$ stellen sind. Mit seiner viel beachteten „Geburtstagszugs-Entscheidung“ vom 13.11.2013 ${ }^{58}$ hat der Bundesgerichtshof seine zum alten Geschmacksmustergesetz vertretene Auffassung aber ausdrücklich aufgegeben. An den Urheberrechtsschutz von Werken der angewandten Kunst seien grundsätzlich keine anderen Anforderungen zu stellen als an den Urheberrechtsschutz von Werken der zweckfreien bildenden Kunst oder des literarischen und musikalischen Schaffens. ${ }^{59}$ Aus dieser Entscheidung folgt aber nicht, dass sämtliche Industriedesigns automatisch Urheberrechtsschutz genießen. Wesentliches Beurteilungskriterium bleibt weiterhin die „künstlerische Leistung“, die in dem Industriedesign zum Ausdruck kommen muss und bei herkömmlichen Produktgestaltungen eher selten gegeben sein dürfte.

\subsubsection{Patentrecht}

Abzugrenzen ist das Designrecht ferner vom Patentrecht, dessen Schutzdauer gem. $\S 16$ PatG 20 Jahre beträgt. Während es im Designrecht um den Schutz der äußeren Gestaltungsformen zwei- oder dreidimensionaler Erzeugnisse und damit sozusagen um die „Hülle“ geht, zielt der Patentschutz auf den Schutz neuartiger technischer oder biotechnischer Erfindungen ab. Die Wirkungen des Patentrechts entstehen gem. $§ 58$ Abs. 1 PatG mit der Veröffentlichung der Erteilung des Patents im Patentblatt.

\footnotetext{
${ }^{54}$ Schulze, in: Eichmann/Kur, $\S \$ 4$ Rn. 3.

${ }^{55}$ BGH, Urteil vom 10.12.1987 - I ZR 198/85, Rn. 22.

${ }^{56}$ Schulze, in: Eichmann/Kur, §§ 4 Rn. 12.

${ }^{57}$ Schulze, in: Eichmann/Kur, $\S \$ 4$ Rn. 14.

${ }^{58} \mathrm{BGH}, \mathrm{NJW} 2014,469 \mathrm{ff}$.

${ }^{59} \mathrm{BGH}, \mathrm{NJW} 2014,469$ (472).
} 


\section{Behörden- und Gerichtsverfahren im nationalen Designrecht}

Mit Inkrafttreten des Designgesetzes zum 01.01.2014 hat auch der Rechtsschutz eine zentrale Neuerung erfahren. Während die Nichtigkeit eines nationalen Designs bis zu diesem Zeitpunkt nur im zivilrechtlichen Klageverfahren überprüft werden konnte, sieht § 34a DesignG nunmehr ein eigenständiges Nichtigkeitsverfahren vor dem Deutschen Patent- und Markenamt vor. Fortan besteht damit die Möglichkeit die Nichtigkeit eines eingetragenen Designs entweder durch Beschluss des Deutschen Patent- und Markenamts oder im Falle einer Widerklage durch Urteil im Verletzungs- oder Schadensersatzverfahren durch das Gericht feststellen oder erklären zu lassen. ${ }^{60}$ Die Voraussetzungen für die Nichtigkeit eines eingetragenen Designs werden in $\S 33$ DesignG geregelt. Differenziert wird zwischen dem Vorliegen absoluter Nichtigkeitsgründe gem. $\S 33$ Abs. 1 DesignG, zu deren Geltendmachung nach $\S 34$ S. 1 DesignG jedermann befugt ist, und den relativen Nichtigkeitsgründen aus $\S 34$ DesignG. ${ }^{61}$ Das Vorliegen eines absoluten Nichtigkeitsgrundes dergestalt, dass es an der Designfähigkeit, der Neuheit oder Eigenart des Designs fehlt oder der Designschutz nach $\S 3$ DesignG ausgeschlossen ist, führt zur Nichtigkeit des eingetragenen Design mit Wirkung ex tunc, ohne dass es einer gesonderten Erklärung bedarf; es genügt die Feststellung. Für den betroffenen Rechtsinhaber besteht bei Vorliegen eines relativen Nichtigkeitsgrundes zudem die Möglichkeit, Antrag auf Erklärung der Nichtigkeit nach $\S \S 33$ Abs. 2, 34 S. 2 DesignG hinsichtlich eines zugunsten eines Dritten eingetragenen Designs zu stellen. Über den Nichtigkeitsantrag entscheidet das Deutsche Patent- und Markenamt gem. § 33 Abs. 3 DesignG durch Beschluss, ${ }^{62}$ gegen den nach $\S 23$ Abs. 4 DesignG die Beschwerde an das Bundespatentgericht statthaft ist. Beschlüsse des Beschwerdesenats des Bundespatentgerichts können schließlich nach $\S 23$ Abs. 5 DesignG mit der Rechtsbeschwerde an den Bundesgerichtshof angefochten werden, sofern die Beschwerde vom Beschwerdesenat zugelassen wurde. Stellt das Deutsche Patent- und Markenamt rechtskräftig die Nichtigkeit eines Designs fest oder erklärt es dessen Nichtigkeit, wird das eingetragene Design gem. § 36 Abs. 1 Nr. 5 DesignG von Amts wegen gelöscht.

Neben dem behördlichen Verfahren vor dem Deutschen Patent- und Markenamt ist bei einer Verletzung des Designschutzes, etwa in der Form einer unbefugten Benutzung eines Designs, der Klageweg zu den ordentlichen Gerichten eröffnet. Für Streitigkeiten in Designrechtsangelegenheiten zwischen Rechtsprätendenten über Unterlassungs- bzw. Beseitigungsansprüche, Schadensersatzansprüche, Auskunftsansprüche, besteht in Deutschland eine ausschließliche sachliche Zuständigkeit der Landgerichte ( $\$ 52$ Abs. 1 DesignG). Die örtliche Zuständigkeit folgt

\footnotetext{
${ }^{60}$ Siehe $\S \S 33$ Abs. 3 DesignG; BT-Drucks. 17/13428, S. 30 f.

${ }^{61}$ BT-Drucks. 17/13428, S. 23.

${ }^{62}$ Ausnahme § 52b DesignG: Zuständigkeit des Designgerichts, wenn die Nichtigkeit eines eingetragenen Designs in Zusammenhang mit einer Klage wegen der Verletzung desselben eingetragenen Designs erhoben wird.
} 
aus $\S § 12$ ZPO. ${ }^{63}$ Parallel zu den allgemeinen Vorschriften der ZPO ist gegen die Urteile des Landgerichts gem. § 511 Abs. 1 ZPO die Berufung zum Oberlandesgericht sowie unter den Voraussetzungen des $§ 566$ Abs. 1 ZPO die Sprungrevision zum Bundesgerichtshof statthaft.

\title{
7 Ausblick
}

Wie die gegenwärtige anhaltende Diskussion in Deutschland um den Ersatzteilmarkt im Automobilsektor zeigt, kann der Designschutz als Investitionsschutz aber auch zu einem ungewollten Wettbewerbshemmnis führen, der sich infolge unangemessener Preise zu Lasten des Verbrauchers auswirkt. Um künftig eine Einschränkung des Designrechts und Öffnung des Marktes für formgebundene Ersatzteile zu erreichen, ${ }^{64}$ sieht der von der Bundesregierung vorgelegte Gesetzesentwurf für ein Gesetz zur Stärkung des fairen Wettbewerbs in $\S 40$ a DesignG die Einführung einer Reparaturklausel für formgebundene Ersatzteile komplexer Erzeugnisse vor, wonach künftig für ein Design, das als Bauelement eines komplexen Erzeugnisses allein zur Reparaturzwecken dieses komplexen Erzeugnisses dient, kein Designschutz besteht. ${ }^{65}$ Aktuell noch offen ist allerdings der konkrete Umfang der Gesetzesänderung, insbesondere die Umsetzung der im Regierungsentwurf derzeit vorgesehenen Stichtagsregelung, ${ }^{66}$ die den schon bestehenden eingetragenen Designs für die Dauer von bis zu 25 Jahren Bestandschutz sichert, was wohl einer eher halbherzigen Reform entspräche.

\footnotetext{
${ }^{63} \mathrm{Vgl}$. Eichmann, in: Eichmann/Kur, §§ 11 Rn. 63.

${ }^{64}$ Vgl. BT-Drucks 19/12084, S. 40.

${ }^{65}$ BT-Drucks. 19/12084, S. 40; zum künftigen Wortlaut des $\S \S 40 a$ DesignG BT-Drucks. 19/12084, S. 17.

${ }^{66} \S \S 73$ Abs. 2 DesignG-E, BT-Drucks. 19/12084, S. 17.
}

\begin{abstract}
Open Access Dieses Kapitel wird unter der Creative Commons Namensnennung 4.0 International Lizenz (http://creativecommons.org/licenses/by/4.0/deed.de) veröffentlicht, welche die Nutzung, Vervielfältigung, Bearbeitung, Verbreitung und Wiedergabe in jeglichem Medium und Format erlaubt, sofern Sie den/die ursprünglichen Autor(en) und die Quelle ordnungsgemäß nennen, einen Link zur Creative Commons Lizenz beifügen und angeben, ob Änderungen vorgenommen wurden.

Die in diesem Kapitel enthaltenen Bilder und sonstiges Drittmaterial unterliegen ebenfalls der genannten Creative Commons Lizenz, sofern sich aus der Abbildungslegende nichts anderes ergibt. Sofern das betreffende Material nicht unter der genannten Creative Commons Lizenz steht und die betreffende Handlung nicht nach gesetzlichen Vorschriften erlaubt ist, ist für die oben aufgeführten Weiterverwendungen des Materials die Einwilligung des jeweiligen Rechteinhabers einzuholen.
\end{abstract}

